\title{
The Potential of Artificial Neural Network Technique in Daily and Monthly Ambient Air Temperature Prediction
}

\author{
Mahboubeh Afzali, Afsaneh Afzali, and Gholamreza Zahedi
}

\begin{abstract}
Ambient air temperature prediction is of a concern in environment, industry and agriculture. The increase of average temperature results in natural disasters, higher energy consumption, damage to plants and animals and global warming. Ambient air temperature predictions are notoriously complex and stochastic models are not able to learn the non-linear relationships among the considered variables. Artificial Neural Network (ANN) has potential to capture the complex relationships among many factors which contribute to prediction. The aim of this study is to develop ANN for daily and monthly ambient air temperature prediction in Kerman city located in the south east of Iran. The mean, minimum and maximum ambient air temperature during the years 1961-2004 was used as the input parameter in Feed Forward Network and Elman Network. The values of $R, M S E$ and MAE variables in both networks showed that ANN approach is a desirable model in ambient air temperature prediction, while the results of one day ahead mean temperature and one month ahead maximum temperature are more precise using Elman network.
\end{abstract}

Index Terms-Artificial neural network, feed forward network, elman network, temperature.

\section{INTRODUCTION}

Air temperatures prediction is of a concern in environment, industry and agriculture. The climate change phenomenon is the main environmental concern in the world threatening the human life on the earth. The industrial activities are so effective in this problem and cause the global warming which the world has been faced with, lately. Knowing the variation of ambient temperature is important in agriculture because extreme changes in air temperature may cause damage to plants and animals [1, 2]. Air temperature forecasting is useful in knowing the probability of tornado, and flood occurrence in an area [3]. Prediction of the energy consumption, soil surface temperature and solar-radiation is related to ambient air temperature forecasting [1].

The prediction of dynamic processes faces many challenges due to continuous changes in affected parameters. Hence, the statistical models are not able to capture the strong nonlinear relationship among different variables in forecasting. ANN models are more flexible and appropriate among the data-based models without needing explicit

Manuscript received December 16, 2011; revised January 4, 2012. Mahboubeh Afzali is with Faculty of Computer Science and Information Systems, Universiti Teknologi Malaysia, Malaysia and Islamic Azad University, Bardsir Branch, Iran (e-mail:afzali mahboobeh@yahoo.com).

Afsaneh Afzali is with Faculty of Chemical Engineering, Universiti Teknologi Malaysia, Malaysia and Young Researchers Club, Islamic Azad University, Kerman Branch, Iran (e-mail: afzali_afsaneh@yahoo.com).

G. Zahedi is with the Chemical Engineering Department, Universiti Teknologi Malaysia, Malaysia (email: grzahedi@cheme.utm..my) mathematical representations. ANN performs better in prediction cases rather than statistical regression analysis $[4,5]$.

Some studies have applied different models in forecasting such as ANN and statistical models [6, 7, 8]. The studies demonstrated that the neural network models outperform multiple linear regression models through handling non-linear associations among variables. ANN has been extensively used for prediction in different applications [9, $10,11,12]$. Smith et al. (2009) found that extreme cold and heat can affect on crops and livestock. They predicted ambient air temperature by use of ANN at time span of 1 to 12 hours. The results showed that ANN is able to forecast air temperature throughout the year with minimum mean absolute error (MAE) and the inclusion of rainfall as input to the model, improves prediction accuracy [2]. In another study the application of back propagation ANN showed desirable results and good accuracy for air temperature prediction even with only one input $[3,13]$.

Dombayc and Golcu (2009) predicted daily mean ambient temperatures by use of an ANN model in Denizli, south-western Turkey. They used the meteorological data of the years 2003-2005 and 2006 as the training and testing data respectively. They analyzed different ANN networks and selected a feed-forward back propagation algorithms consists of 3 inputs, 6 hidden neurons and 1 output [1]. Behrang et al (2010) developed Multi-layer perceptron (MLP) and radial basis function (RBF) neural networks for daily global solar radiation prediction. They considered various meteorological variables including Daily mean air temperature, relative humidity, sunshine hours, evaporation, and wind speed values between 2002 and 2006 for Dezful city in Iran. The results of comparison between $\mathrm{ANN}$ and other prediction methods showed ANN suitability in prediction due to less Mean Absolute Percentage Error (MAPE) [14].

The objective of this paper is to predict the maximum minimum and mean ambient air temperature developing ANN models for one day and one month ahead. Continuous temperature data for 44 years for the case study of Kerman City, Iran were used in train, validation and test phases of the ANN models. While the maximum, minimum and mean temperature values of 1961- 1995 were used in train and validation phases, the values of the year 1996- 2004 were used in the test phase of the ANN models. The Feed Forward and Elman networks have been used in different exercises based on the maximum, minimum and mean ambient air temperature as inputs. The suitability of the preferred networks has been interpreted based on the correlation coefficient (R) and some statistical criteria. In section 2 of the paper, data set used in the research and artificial neural 
network approach is presented in more details, fallowing with the results and discussion section. Finally the conclusion is drawn in Section 4.

\section{MATERIALS AND METHODS}

\section{A. Data Sets}

The data used in this study are daily and monthly mean, minimum and maximum ambient air temperature related to Kerman city in the south east of Iran. These data were collected from Meteorological Organization of Kerman Province. Ambient air temperature is based on degree centigrade. The collected data are corresponded to forty four years period from 1 January 1961 to 31 December 2004. The data were considered in three different data sets including train, validation and test in artificial neural network. The average of minimum, mean and maximum temperature of forty-four years based on months is shown in Fig. 1.

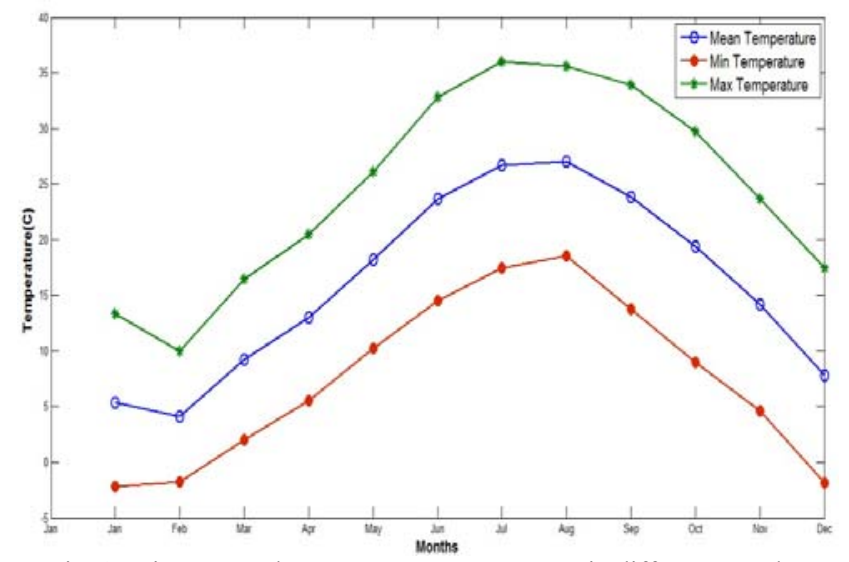

Fig. 1. Min, max and mean average temperature in different months.

\section{B. Artificial Neural Network Approach}

ANN is based on the neurons connected or functionally-related to each other; imitate the behavior of human biological neurons. The extensive applications of neural networks are pattern recognition and classification, time series prediction and modeling [15]. Neurons which are the basic components of the neural network are interconnected through different layers such as input, hidden and output layers. The degree of interconnection is defined by the weight which means the impact of neuron on neuron. The structure of neural network is shown in Fig. 2.

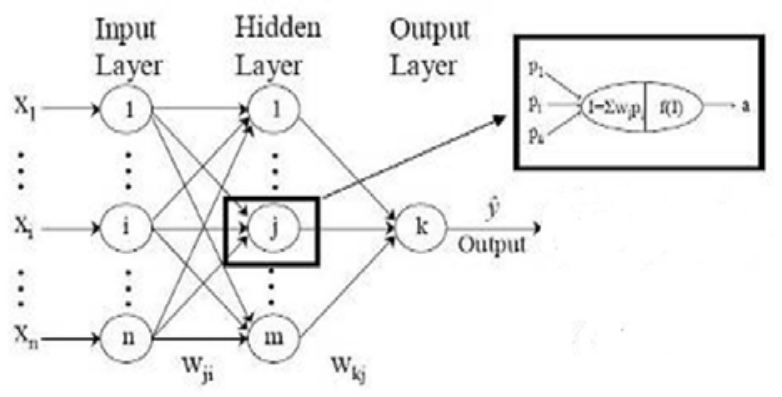

Fig. 2. Schematic of a neural network

Each neuron is activated by an activation function based on a threshold value. The transfer activation is processed by both input signals neighbor nodes belonging to different layers and output signal such as bias to get the output of neuron. The common transfer functions in the ANN are tangent sigmoid (tansig) function and linear function (pureline) depicted respectively in Fig. 3. The formula of tansig and pureline transfer functions are expressed respectively in equation 1 and 2 :

$$
\begin{gathered}
F_{k}\left(s_{k}\right)=\frac{2}{1+e^{\left(-2 s_{k}\right)}-1} \\
F_{k}\left(s_{k}\right)=s_{k}
\end{gathered}
$$

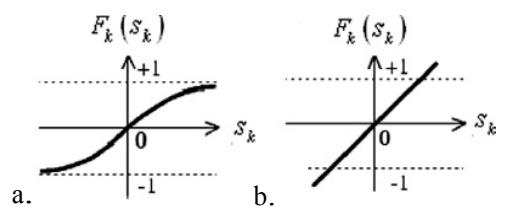

Fig. 3. Transfer function a. tansig and b. pureline[15].

The important factor on performance is the topology of ANN. ANN is classified into major groups based on the pattern of interconnection of neurons to propagate data. The main categories of neural networks are Feed Forward and Recurrent Neural Networks. In Feed Forward Networks such as Multi Layer Perceptron (MLP) and Feed Forward Network (FNN), data enter into input layer for processing layer by layer to achieve the output layer. Recurrent Neural Networks such as Elman and Hopfield have the recurrent path through feed backing from output layer to hidden layer for the detection and generation of time-varying patterns. ANN should be configured to produce the desired output by adjusting the weights of interconnections among all neuron pairs. This process is called as training which is categorized into two main groups called supervised and unsupervised learning. In supervised learning, ANN feeds with the learning patterns and adjusts the weights by comparison of the desired output with the actual output obtained from the input variables to achieve the minimum error. On the contrary, unsupervised learning is based on discovering the features of input data in a statistical manner by input irritant classification [15]. In this paper, we focus on the supervised learning. The most popular learning used in the prediction purpose is the Back Propagation Algorithm (BPA) which aims to reduce the overall system error.

\section{RESUltS AND DISCUSSION}

Two types of neural network have been applied in two scenarios. One network is the FNN and the next network is Elman. The tansig and pureline functions were used for the neurons in the hidden layer and output layer, respectively. The input and target values were normalized into the range of $[-1,1]$ in the pre-processing phase [16]. The weights and biases were adjusted based on the Levenberg-Marqardt optimization (trainlm). The Mean Square Error (MSE) and Mean Average Error (MAE) were chosen as the statistical criteria for measuring of the network performance.

Mean, minimum and maximum air temperatures were considered as input and output of the network. Three 
different data sets were extracted from the input and target data for train, validation and test phases. While training set consists of 50 percent of data to build the model and determine the parameters such as weights and biases, validation data set includes 25 percent to measure the performance of network by holding constant parameters. Finally, 25 percent of data is used to increase the robustness of model in the test phase.

The validation and test phases are very important due to misleading of small error in the training phase. If the network is not trained well due to the irrelevant data of the individual cases such as over fitting, it leads to the small error in the training set and makes large error during validation and test phases. While the purpose of training phase is based on learning, it is not a good metric for the performance of network in validation phase.

The overview of the parameters and their values in two different scenarios are depicted in Table I. It can be seen from the table that the two applied neural networks have small errors in predicting ambient air temperature values. The predication of daily mean temperature using Elman network shows better agreement between the predicted and observed values rather than other exercises.

As shown in Fig. 4 and 5, there is a good agreement between predicted and observed values, while the correlation coefficient is 0.97 and 0.98 for one day ahead and 0.78 and 0.80 for one month ahead mean temperature using FNN and Elman networks, respectively. Fig. 6 and 7 also illustrates this matter for minimum temperature value. Fig. 8 and 9 show the agreement in the similar way for maximum temperature.

Temporal variations of the predicted and observed mean temperature values for one day ahead during 360 days using Elman networks are shown in Fig. 10. According to figure 10, the mean temperature values during summer period are higher than winter period due to strong sun light intensity.

TABLE I: STRUCTURE AND TRAINING RESULTS FOR THE NEURAL NETWORK MODELS.

\begin{tabular}{|c|c|c|c|c|c|c|c|c|c|}
\hline Net Type & Topology & Training function & Learning & Learning rate & MSE & MAE & $\mathrm{R}$ & Epoch & Use \\
\hline FNN & 15,1 & Tangsig-purelin & trainlm & 0.01 & 0.004 & 0.049 & 0.973 & 35 & Daily Mean \\
\hline FNN & 15,1 & Tangsig-purelin & trainlm & 0.01 & 0.008 & 0.071 & 0.939 & 54 & Daily Min \\
\hline FNN & 15,1 & Tangsig-purelin & trainlm & 0.01 & 0.008 & 0.067 & 0.966 & 130 & Daily Max \\
\hline Elm & 15,1 & Tangsig-purelin & trainlm & 0.01 & 0.004 & 0.049 & 0.977 & 35 & Daily Mean \\
\hline Elm & 15,1 & Tangsig-purelin & trainlm & 0.01 & 0.008 & 0.690 & 0.942 & 57 & Daily Min \\
\hline Elm & 15,1 & Tangsig-purelin & trainlm & 0.01 & 0.008 & 0.064 & 0.967 & 13 & Daily Max \\
\hline FNN & 15,1 & Tangsig-purelin & trainlm & 0.01 & 0.083 & 0.223 & 0.715 & 15 & Monthly Min \\
\hline FNN & 15,1 & Tangsig-purelin & trainlm & 0.01 & 0.064 & 0.201 & 0.80 & 91 & Monthly Max \\
\hline Elm & 15,1 & Tangsig-purelin & trainlm & 0.01 & 0.069 & 0.213 & 0.80 & 16 & Monthly Mean \\
\hline Elm & 15,1 & Tangsig-purelin & trainlm & 0.01 & 0.081 & 0.228 & 0.734 & 11 & Monthly Min \\
\hline Elm & 15,1 & Tangsig-purelin & trainlm & 0.01 & 0.060 & 0.193 & 0.811 & 11 & Monthly Max \\
\hline
\end{tabular}
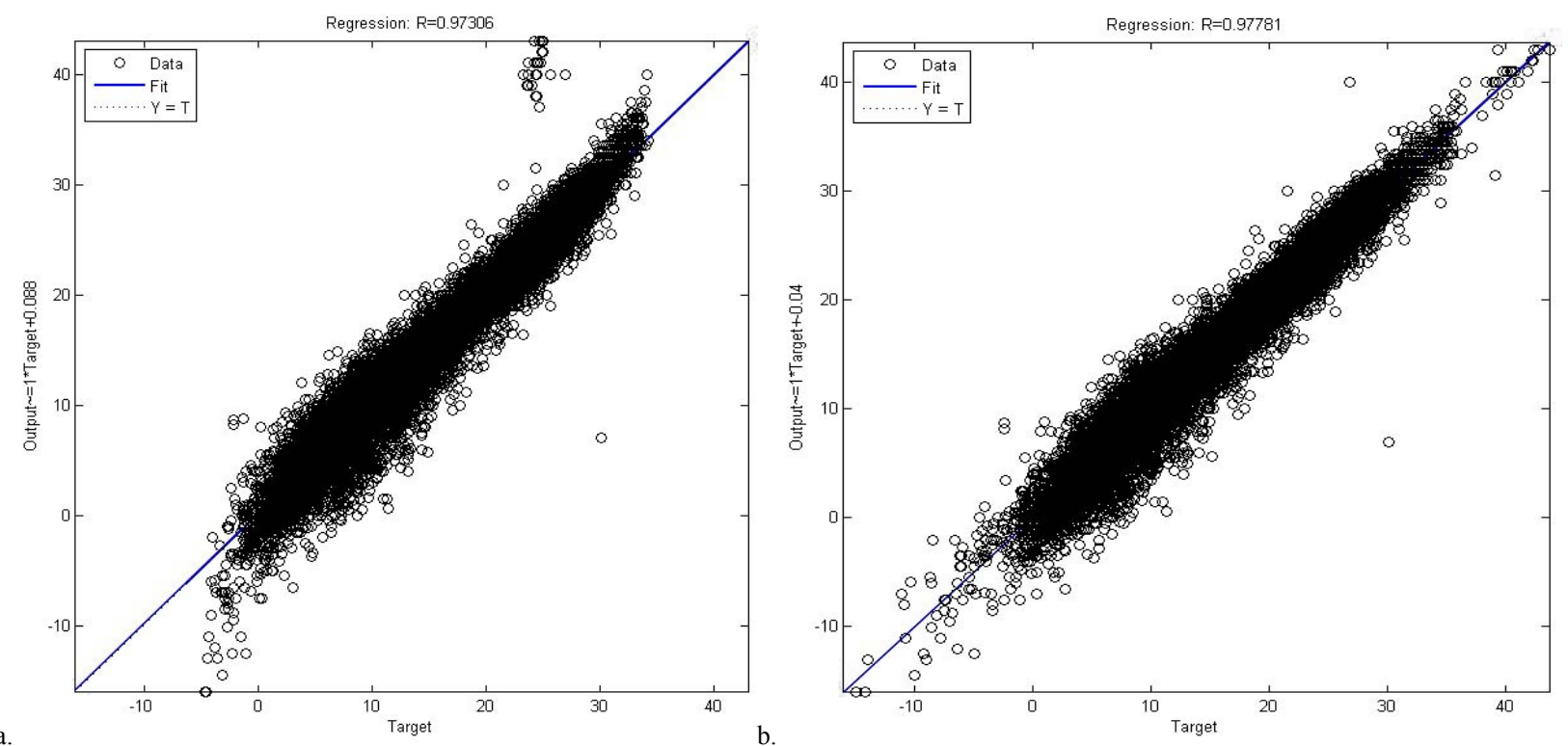

Fig 4. Comparison between the daily predicted and estimated mean temperature using a.FNN and b.Elman network 

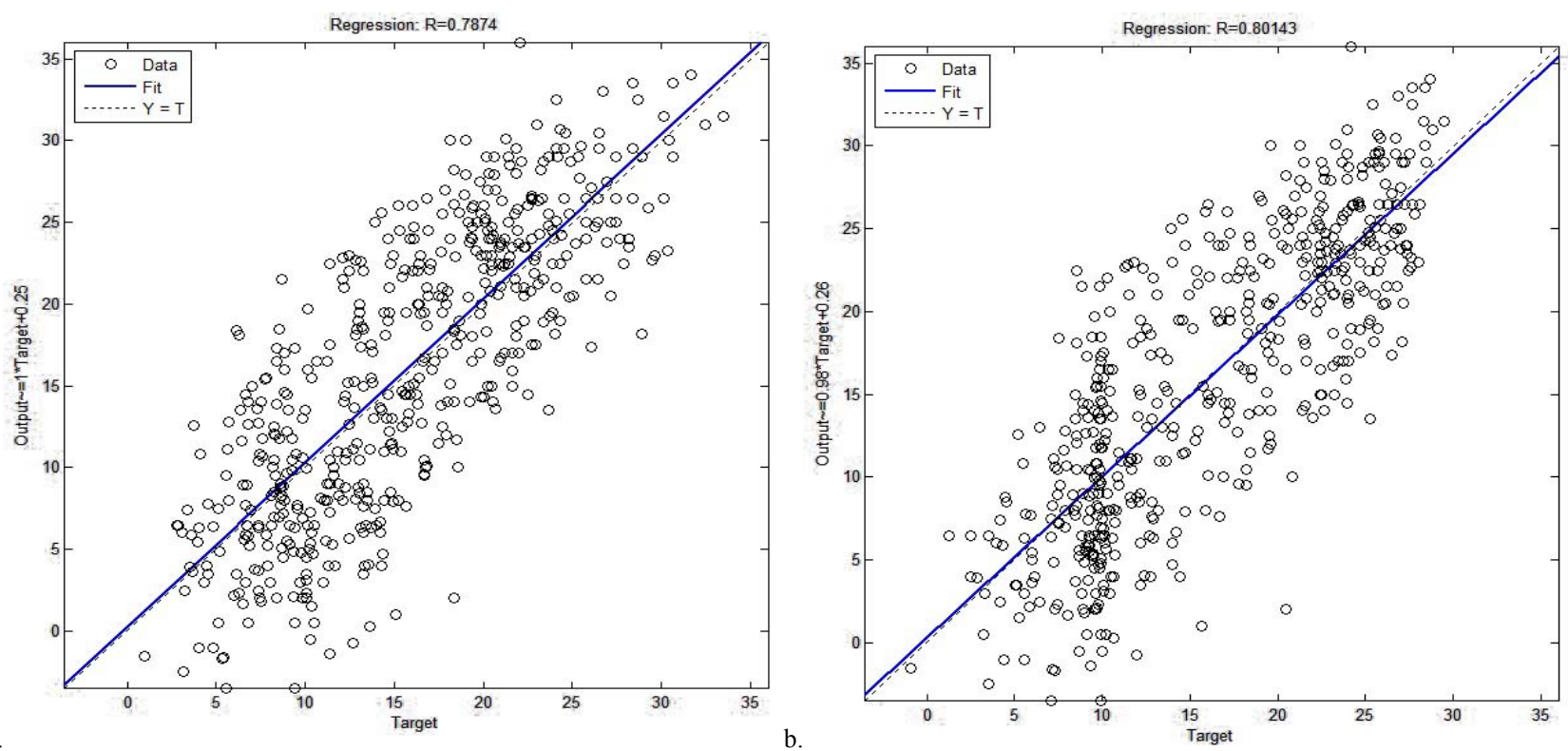

Fig 5. Comparison between the monthly predicted and estimated mean temperature using a.FNN and b.Elman network
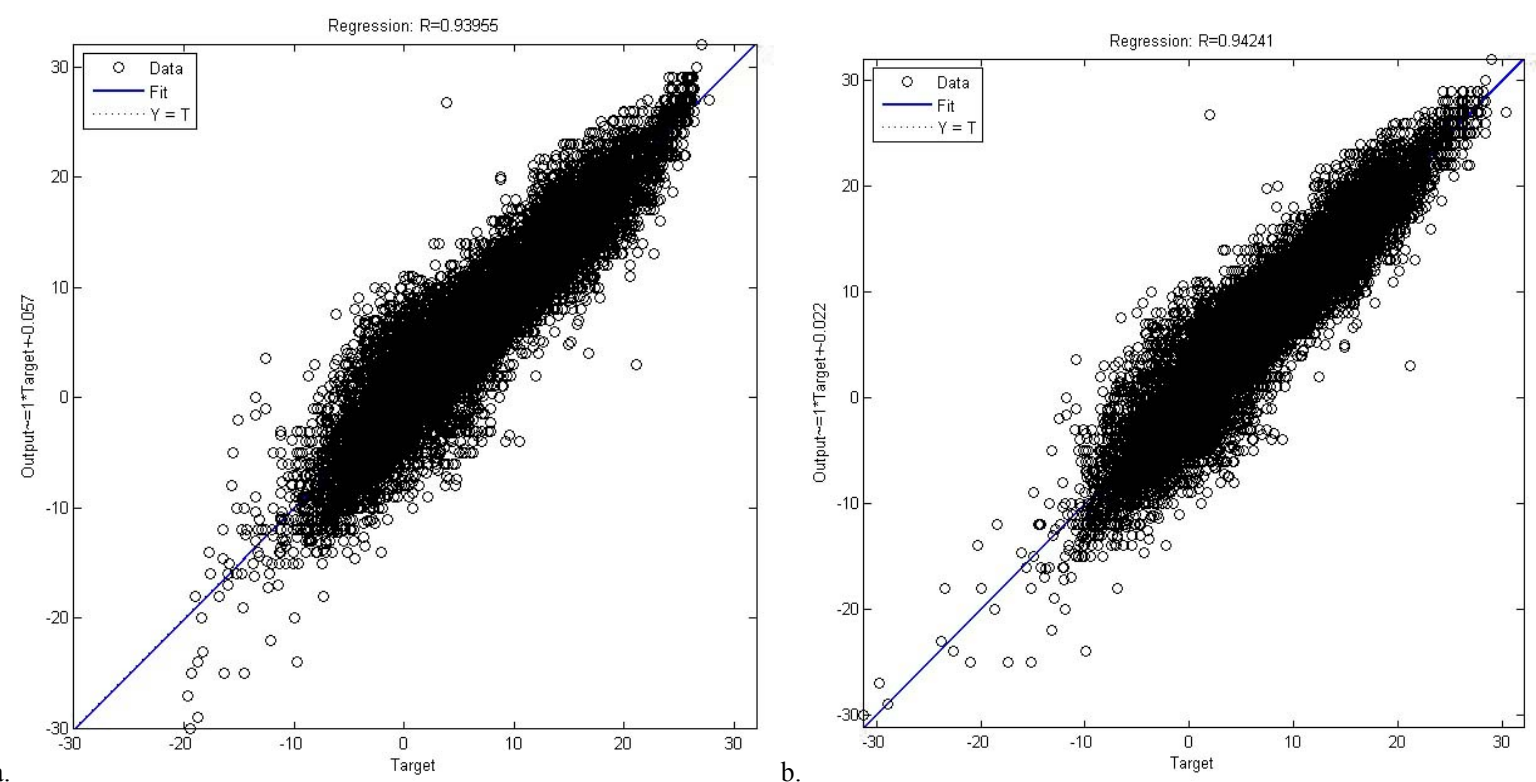

Fig 6. Comparison between the daily predicted and estimated minimum temperature using a.FNN and b.Elman network
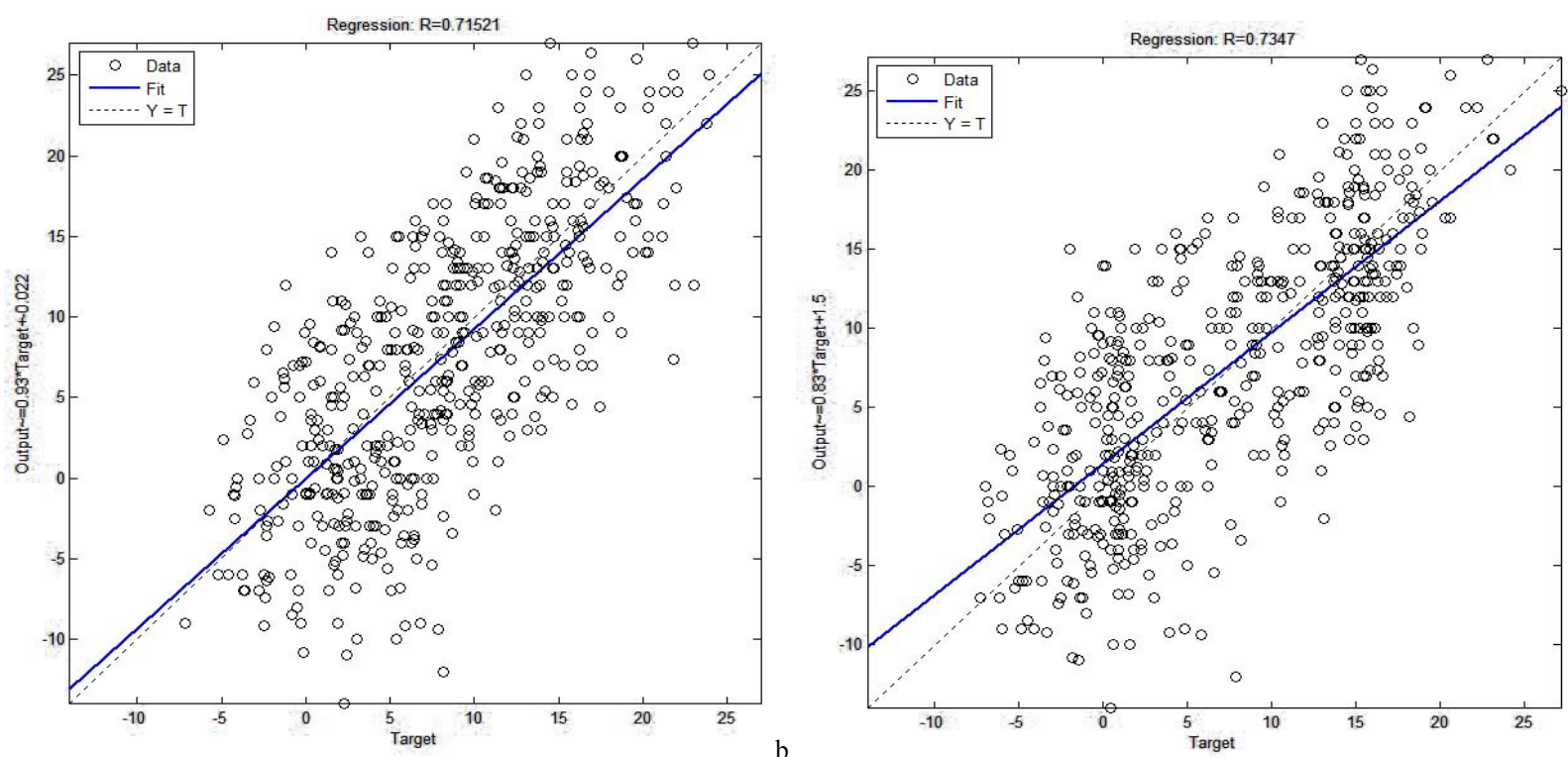

Fig 7. Comparison between the monthly predicted and estimated minimum temperature using a.FNN and b.Elman network 

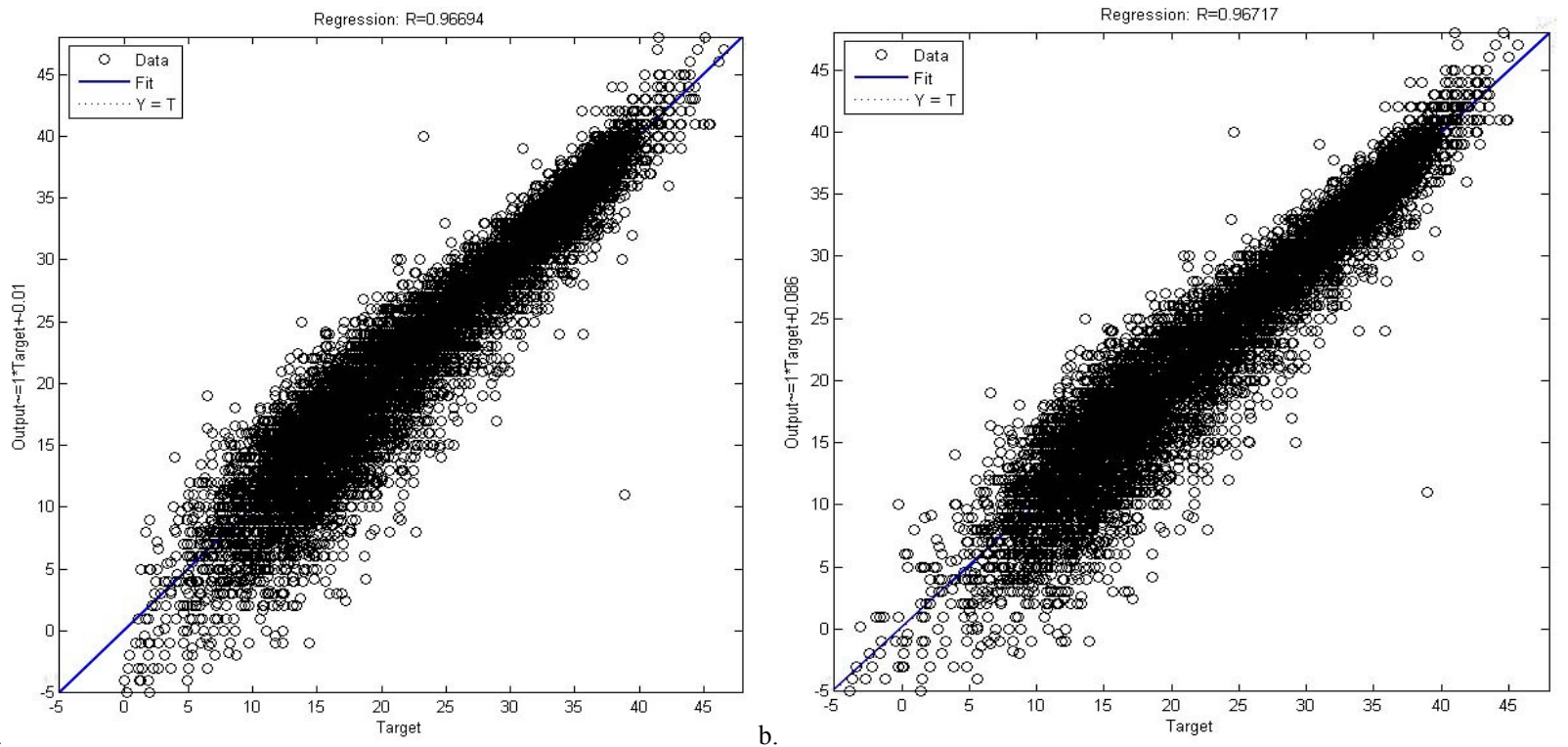

Fig 8. Comparison between the daily predicted and estimated maximum temperature using a.FNN and b.Elman network
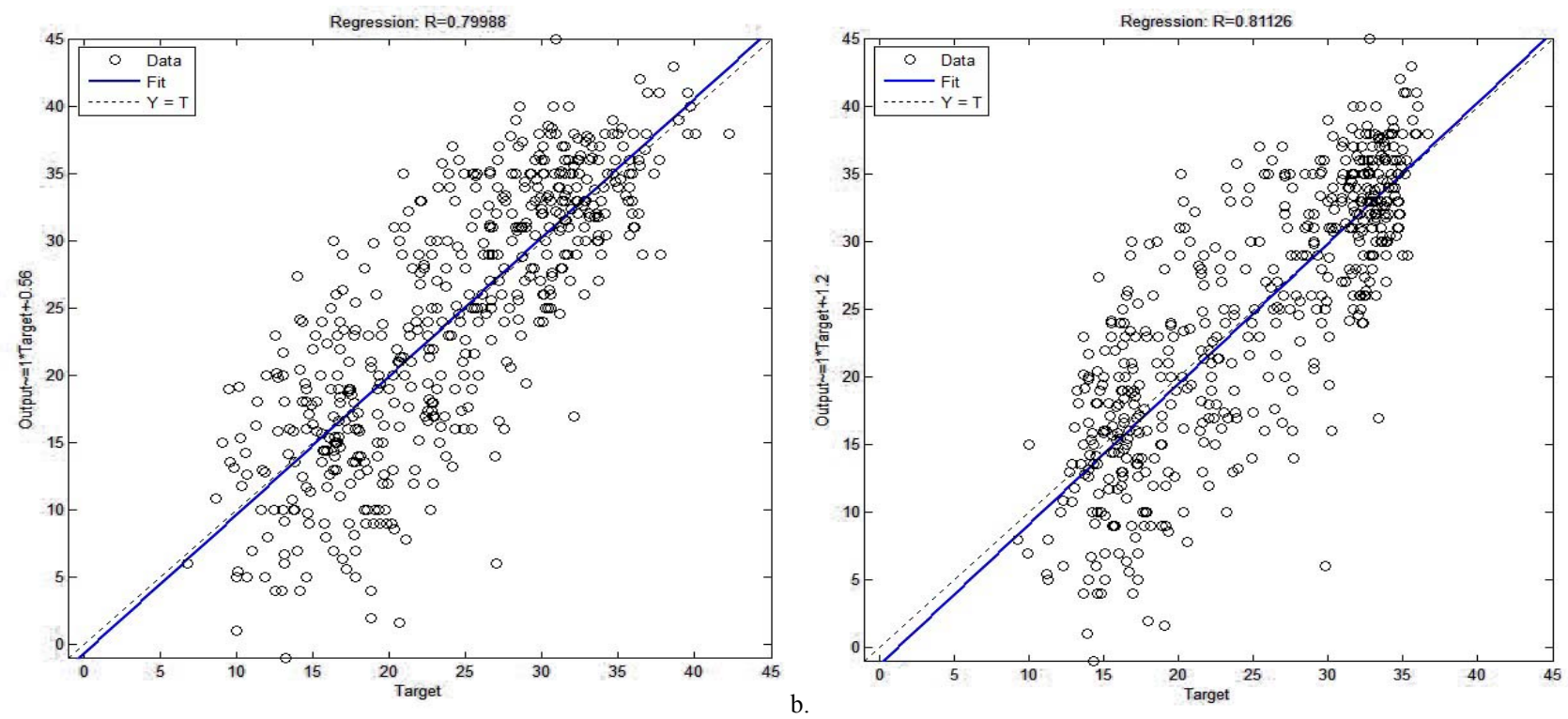

Fig 9. Comparison between the monthly predicted and estimated maximum temperature using a.FNN and b.Elman network.

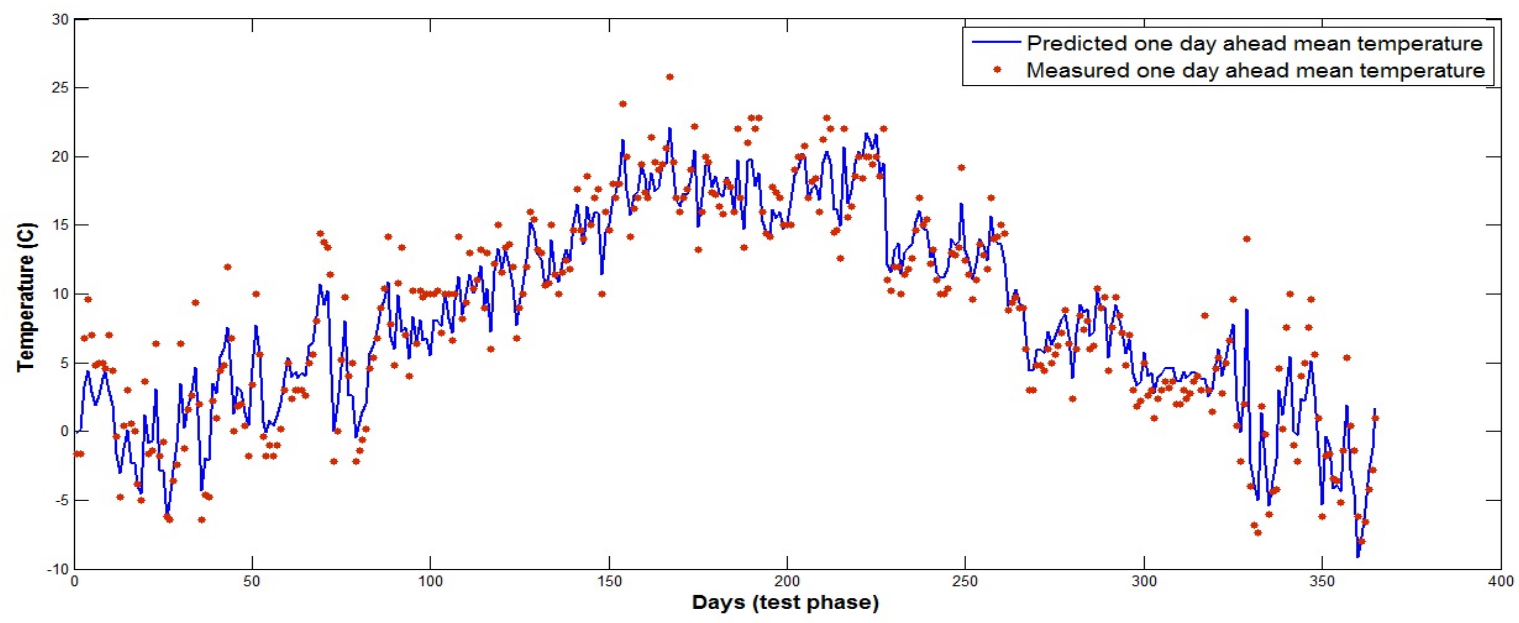

Fig 10. Comparison between one day ahead predicted and observed mean temperature value using.Elman network in 2004. 


\section{CONCLUSION}

Two different FNN and Elman neural networks have been developed in this study to predict the daily and monthly mean, minimum and maximum ambient air temperature in Kerman city, Iran. Mean, minimum and maximum air temperature ware considered as inputs in the networks for the time series of the year 1961-2004. The output of the models is composed of one day and one month ahead air temperature prediction. MSE and MAE statistical indicators showed small values that demonstrates the correct behavior of the developed forecasting models. The values of the correlation coefficient demonstrate the good agreement between predicted and observed values while the results from Elman network are more precise than FNN network. Hence, neural networks are robust models for predicting meteorological condition parameters in weather management systems.

\section{REFERENCES}

[1] R. J. Vidmar. (August 1992), "On the use of atmospheric plasmas as electromagnetic reflectors,” IEEE Trans. Plasma Sci. [Online]. 21(3). pp. 876-880. Available: http://www.halcyon.com/pub/journals/21ps03-vidmar

[2] O. A. Dombayc, and M. Golcu, "Daily means ambient temperature prediction using artificial neural network method: A case study of Turkey," Renewable Energy. 2009, 34: pp.1158-1161.

[3] B. A. Smitha. G. Hoogenbooma. R. W. McClendon, "Artificial neural networks for automated year-round temperature prediction," Computers and Electronics in Agriculture. 2009, 68: pp.52-61.

[4] I. Tasadduq. S. Rehman. K. Bubshait, "Application of neural networks for the prediction of hourly mean surface temperatures in Saudi Arabia," Renewable Energy. 2005, 25: pp.545-554.

[5] K. S. Yigit. H. M. Ertunc, "Prediction of the air temperature and humidity at the outlet of a cooling coil using neural networks," International Communications in Heat and Mass Transfer. 2006, 33: pp. 898-907.

[6] G. B. Sahoo. S. G. Schladow. J. E. Reuter, "Forecasting stream water temperature using regression analysis, artificial neural network, and chaotic non-linear dynamic models," Journal of Hydrology. 2009, 378: pp.325-342.

[7] U. Schlink. O. Herbarth. M. Richter. S. Dorling. G. Nunnari. G. Cawley. E. Pelikan, "Statistical models to assess the health effects and to forecast ground-level ozone," Environmental Modelling \& Software. 2006, 21: pp.547-558.

[8] M. Cai. Y. Yin. M. Xie, "Prediction of hourly air pollutant concentrations near urban arterials using artificial neural network approach," Transportation Research. 2009, 14(Part D), pp. 32-41

[9] J. C. M. Pires. F. G. Martins, "Correction methods for statistical models in tropospheric ozone forecasting," Atmospheric Environment. 2011, 45: pp. 2413-2417.

[10] D. Jianga. Y. Zhanga. X. Hua. Y. Zenga. J. Tanb. D. Shao, "Progress in developing an ANN model for air pollution index forecast," Atmospheric Environment. 2004, 38: pp.7055-7064.
[11] G. Grivas. A. Chaloulakou, "Artificial neural network models for prediction of PM10 hourly concentrations, in the Greater Area of Athens," Greece. Atmospheric Environment. 2006, 40: pp.1216-1229.

[12] M. L. Martin. I. J. Turias. F. J. Gonzalez. P. L. Galindo. F. J. Trujillo. C. G. Puntonet. J. M. Gorriz, "Prediction of CO maximum ground level concentrations in the Bay of Algeciras, Spain using artificial neural networks," Chemosphere. 2008, 70: pp.1190-1195.

[13] S. Palani. P. Tkalich. R. Balasubramanian. J. Palanichamy, "ANN application for prediction of atmospheric nitrogen deposition to aquatic ecosystems," Marine Pollution Bulletin. 2011, in press.

[14] Zahedi, G., S. Mohammad Zadeh, "A new vapor pressure equation for pure substances, Korean Journal of Chemical Engineering," 25, 6, 1514-1517, 2008.

[15] M. A. Behrang. E. Assareh. A. Ghanbarzadeh. A. R. Noghrehabadi, "The potential of different artificial neural network (ANN) techniques in daily global solar radiation modeling based on meteorological data," Solar Energy. 2010, 84: 1468-1480.

[16] S. Haykin,. "Neural networks. a comprehensive foundation." Upper Saddle River, NJ: Prentice-Hall, 1999.

[17] H. Demuth. M. Beale. M. Hagan, "Matlab neural network toolbox user's guide, version 5.1," The MathWorks Inc, 2007.

Mahboubeh Afzali is a $\mathrm{PhD}$ candidate at the Faculty of Computer Science and Information Systems, Universiti Teknologi Malaysia (UTM), Skudai, Malaysia. She received the BSc degree in Computer Engineering from Shahid Bahonar University, Kerman, Iran, in 2004, the MSc degree in Computer System Architecture Engineering from Islamic Azad University, Arak Branch, in 2007. Her research interests are in the areas of wireless network, fault tolerance and neural network.

Afsaneh Afzali is a PhD candidate at the Faculty of Chemical Engineering, Universiti Teknologi Malaysia (UTM), Skudai, Malaysia. She received the BSc degree in Environmental Science, Natural Resources Engineering from Yazd University, Yazd, Iran, in 2006, the MSc degree in Environmental Science, Natural Resources Engineering from Isfahan University of Technology, in 2009. Her research interests are in the areas of air pollution, solid waste management, site selection, GIS and neural network.

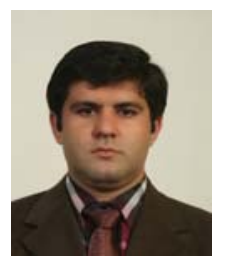

Dr. Gholamreza Zahedi is associate professor in Faculty of Chemical Engineering, Universiti Teknologi Malaysia (UTM), Skudai, Malaysia. He has BSc in Chemical Engineering from Sharif Un. of Technology, Tehran, Iran and MSc and PhD from Shiraz Un., Shiraz, Iran. He is working in process system engineering for period of 6 years after his $\mathrm{PhD}$. He has been also one year in Un. of Waterloo, Canada for post doctorate research. Dr Zahedi has more than 150 publications including papers, conferences, books, and book chapters.

$\mathrm{He}$ is a chartered engineer, First Malaysian foreigner energy manger, Associate editor of Research Journal of Applied Sciences, Engineering and Technology, International Journal of Environment and Chemical Engineering, and technical member of International Association of Science and Technology for Development for 10 years. He is conducting workshops, teaching and consultancy on modeling and optimization for university and industry. 